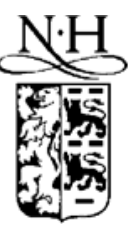

\title{
The effect of protons on the performance of swept-charge devices
}

\author{
David R. Smith ${ }^{\mathrm{a}^{*}}$, Jason Gow ${ }^{\mathrm{a}}$ \\ ${ }^{a}$ Imaging for Space and Terrestrial Applications Group, School of Engineering and Design, \\ Brunel University, Uxbridge, Middlesex, UB8 3PH, UK
}

Elsevier use only: Received date here; revised date here; accepted date here

\begin{abstract}
The e2v technologies CCD54, or swept-charge device (SCD) has been extensively radiation tested for use in the Chandrayaan-1 X-ray Spectrometer (C1XS) instrument, to be launched as part of the Indian Space Research Organisation (ISRO) Chandrayaan-1 payload in 2008. The principle use of the SCD is in X-ray fluorescence (XRF) applications, the device providing a relatively large collecting area of $1.1 \mathrm{~cm}^{2}$, and achieving near Fano-limited spectroscopy at $-15^{\circ} \mathrm{C}$, a temperature that is easily obtained using a thermoelectric cooler (TEC). This paper describes the structure and operation of the SCD and details the methodology and results obtained from two proton irradiation studies carried out in 2006 and 2008 respectively, to quantify the effects of proton irradiation on the operational characteristics of the device. The analysis concentrates on the degradation of the measured FWHM of various elemental lines and quantifies the effects of proton fluence on the observed X-ray fluorescence (XRF) spectra from mineralogical target samples. (C) 2008 Elsevier Science. All rights reserved
\end{abstract}

Keywords: radiation damage; proton; swept-charge device; SCD; XRF

\section{Introduction}

For the use of any charge coupled device (CCD) in space it is necessary to have a thorough understanding of the space radiation environment the device will be subjected to and the resulting effects of the radiation environment on the performance of the device. To this end, two proton irradiation studies have been carried out in support of the Chandrayaan-1 X-ray Spectrometer (C1XS) instrument to be flown on the Indian Space Research
Organisation (ISRO) Chandrayaan-1 lunar mission in late 2008 [1].

The first study was carried out at the Kernfysisch Versneller Instituut (KVI) in 2006 and involved the irradiation of two swept-charge devices (SCDs) to $10 \mathrm{MeV}$ equivalent proton fluencies of $2.1 \times 10^{8}$ and $4.3 \times 10^{8}$ protons. $\mathrm{cm}^{-2}$. At the time of the study these fluence values represented approximately $42 \%$ and $86 \%$ of the expected end-of-life (EOL) total proton fluence respectively [2]. Following the completion of this study, the C1XS instrument design was modified slightly to improve the level of radiation shielding around the SCD detectors. This change, coupled with the launch date moving from the planned April 2008

\footnotetext{
${ }^{*}$ Corresponding author. Tel.: +44 (0)1895 266593; fax: +44 (0)1895 269773; e-mail: david.smith@brunel.ac.uk.
} 
to later in the year, necessitated a second proton irradiation study to obtain an understanding of the effects of the revised EOL proton fluence on the operation of the SCDs. Combining the results from each of the two studies allows for a greater understanding of how the devices will function throughout the operation of C1XS and also shows how SCD device performance varies over a wide range of proton irradiation levels for their possible application in other areas.

This paper will now give a brief overview of the SCD before describing the 2008 proton irradiation study and comparing the collected data sets with those obtained in 2006.

\section{The swept-charge device (SCD)}

The CCD54 SCD detectors were developed by $\mathrm{e} 2 \mathrm{v}$ technologies with funding from the Particle Physics and Astronomy Research Council (now STFC) and the Department of Trade and Industry as part of the IMPACT programme [3]. The device benefits from a large detection area of $1.1 \mathrm{~cm}^{2}$ and achieves near Fano-limited spectroscopy at $-15^{\circ} \mathrm{C}$, a temperature that is easily achievable using a thermoelectric cooler (TEC). The active area is covered with 1725 diagonal electrodes with the isolation channels in the underlying silicon arranged in a herringbone structure, as shown in Fig. 1. The pitch of the channel stops is $25 \mu \mathrm{m}$.

The SCD is read out in a similar manner to a conventional $\mathrm{CCD}$, requiring 575 clock triplets to read-out the whole device area, the charge in each diagonal element having to move through the same number of clock cycles to reach the read-out node. Unlike a conventional CCD, the output from the SCD is a single row of 575 samples, rather than a $2 \mathrm{D}$ image. The charge packets can either be reset per sample, or can be integrated with a reset every n-samples. These two different modes of operation were used to characterise the SCDs used in the presented study. A more detailed description of the SCD and its operation can be found in [4].

In the presented study, the energy resolution of the SCD devices was the most important parameter to be investigated both before and after proton irradiation. For the purposes of X-ray emission line full width at half maximum (FWHM) calculations, only isolated $\mathrm{X}$-ray events were considered in the analysis.

The SCD detectors used in the presented study were initially developed for use in the Demonstration of a Compact Imaging X-ray Spectrometer (D-CIXS) instrument onboard the European Space Agency (ESA) SMART-1 mission and are arranged in a group of four devices on one ceramic package or 'module' $[5,6]$.

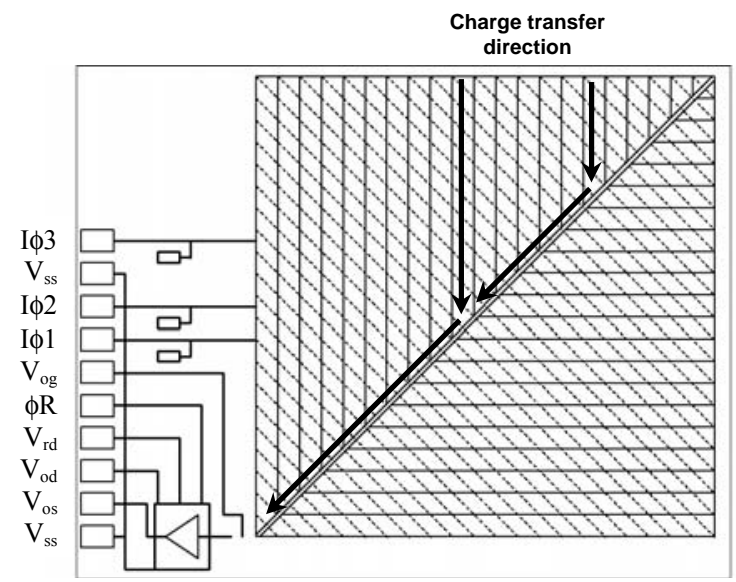

Fig. 1. A schematic of the SCD showing the diagonal electrodes (dashed lines) and underlying isolation channels (solid lines) arranged in a herringbone structure. The charge transfer direction during read out of the device is indicated by the arrows.

\section{SCD characterisation}

The SCDs were characterised in a vacuum chamber test facility both before and after proton irradiation, the device under test being mounted on a shapal (aluminium nitride ceramic) cold finger attached to a TEC. The TEC was in-turn mounted on a copper block with internal water cooling. Temperature control to $\pm 0.1{ }^{\circ} \mathrm{C}$ was provided by a Lakeshore controller (model number 331), the temperature being monitored by a $1000 \Omega$ PRT attached to the side of the ceramic SCD package. Data were recorded at temperatures ranging from $-40{ }^{\circ} \mathrm{C}$ to $+10{ }^{\circ} \mathrm{C}$ using two different operational modes.

The first mode, Mode 1, was used to read-out the whole device area, resetting the charge packet for each subsequent sample, allowing the FWHM of 
various characteristic X-rays emission lines and the device noise to be measured.

The second operational mode, Mode 2, was used to integrate the charge, using a programmable delay between successive line read-outs of between $1 \mathrm{~ms}$ to $15 \mathrm{~s}$. This mode allowed the characteristic SCD triangular leakage current profile to be obtained (the slope being caused by the changing size of the sample collection area) [2, 4].

Attached to one side of the test facility was a tungsten filament Oxford Instruments X-ray tube positioned to illuminate a rotatable target wheel within the chamber, the resulting fluoresced X-rays being incident on the SCD being characterised.

SCD clock and bias voltages were provided by Xcam Ltd. CCD drive electronics and the data were recorded on a laptop computer.

\section{Proton irradiation methodology}

Following the 2006 SCD proton irradiation study, changes were made in the C1XS instrument design to incorporate an additional $1 \mathrm{~mm}$ of aluminium in the instrument structure surrounding the SCD detectors, increasing the level of radiation shielding. There has also been a delay in the original launch date of April 2008 to September 2008, pushing the mission into a period of higher solar activity. These factors were incorporated into a new radiation environment analysis using the ESA Space Environment Information System (SPENVIS) [7] to obtain the revised EOL $10 \mathrm{MeV}$ equivalent proton fluence for C1XS.

The 2008 proton irradiations were carried out at the University of Birmingham using their MC40 cyclotron. The $15 \mathrm{MeV}$ protons emitted were attenuated by a $30 \mu \mathrm{m}$ havar window (allowing the beam to exit into air), a $100 \mu \mathrm{m}$ tungsten scattering foil (to make the beam flux more uniform over the target area), the air between the beam exit and the target SCD and the presence of Gafchromic film (positioned in front of the target SCD to measure the proton fluence received). The SCDs were positioned in an aluminium holder at a distance from the beam exit corresponding to an attenuated proton energy of $10 \mathrm{MeV}$ incident at the surface of the SCD.
Table 1

Summary of proton irradiation parameters

\begin{tabular}{lll}
\hline $\begin{array}{l}10 \mathrm{MeV} \text { equivalent } \\
\text { proton fluence } \\
(\text { protons.cm }\end{array}$ & $\begin{array}{l}\text { Percentage of total } \\
\text { EOL flounce }\end{array}$ & $\begin{array}{l}\text { Irradiation } \\
\text { duration } \\
(\%)\end{array}$ \\
\hline $7.5 \times 10^{8}$ & 100 & 15 \\
$4.3 \times 10^{8}$ & $57^{\mathrm{a}}$ & 2 \\
$3.0 \times 10^{8}$ & 40 & 7.5 \\
$2.1 \times 10^{8}$ & $28^{\mathrm{a}}$ & 2
\end{tabular}

${ }^{\mathrm{a}} 2006$ study data.

The beam flux rate at the target distance was calibrated prior to each irradiation using Gafchromic film (HD810), allowing the calculation of the time required to reach the target fluences. Beam flux uniformity was found to be within $10 \%$ across the SCD target area. The irradiations were carried out with the SCDs un-biased, the whole detector area being irradiated in each case.

Table 1 shows the proton fluencies given to the two SCDs irradiation in the 2008 study, along with those from the 2006 study, indicating the corresponding percentage of the 2008 calculated EOL fluence they each represent.

\section{Post-irradiation analysis}

Fig. 2 shows recorded leakage current profiles from the SCD irradiated to $7.5 \times 10^{8}$ protons.cm ${ }^{-2}$ both before and after proton irradiation at $-20{ }^{\circ} \mathrm{C}$. The characteristic triangular SCD leakage current profile is symmetrical following irradiation, demonstrating a uniform proton irradiation across the device.

Fig. 3 shows the effect of increasing $10 \mathrm{MeV}$ equivalent proton fluence on the measured FWHM of $\mathrm{Mn}-\mathrm{K} \alpha \mathrm{X}$-rays vs. temperature. Data from both the 2008 and 2006 irradiation studies are shown, revealing a proportional increase in measured FWHM for a given increase in proton fluence.

Fig. 4 shows the recorded XRF spectrum from a sample of lunar regolith simulant (JSC-1A) at $-20{ }^{\circ} \mathrm{C}$ both prior to irradiation and following proton irradiation to $40 \%$ and $100 \%$ of the expected $\mathrm{C} 1 \mathrm{XS}$ EOL proton fluence. 


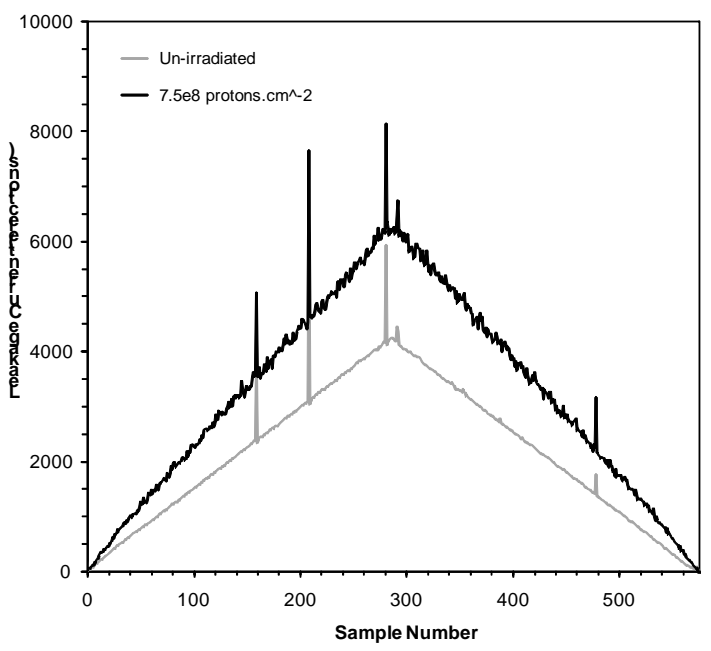

Fig. 2. SCD leakage current profiles.

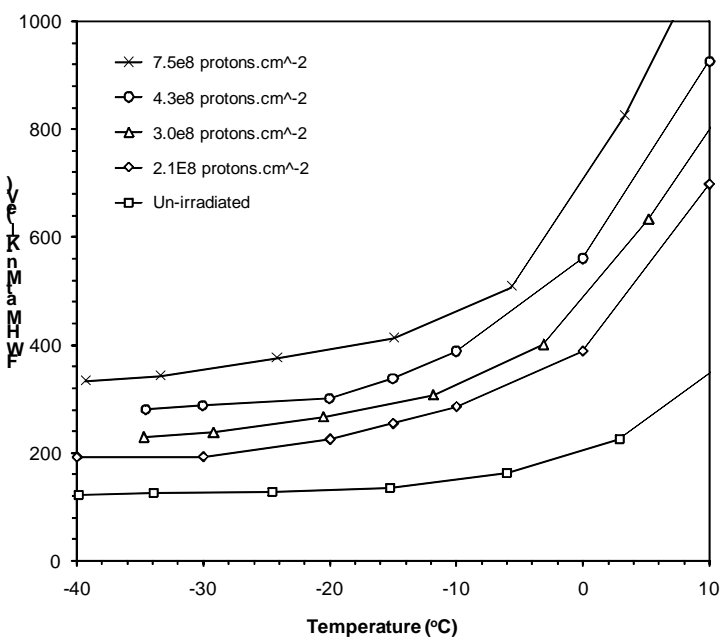

Fig. 3. FWHM of Mn-K $\alpha$ at different operating temperatures for different levels of proton irradiation.

\section{Conclusion}

After proton irradiation to a fluence representative of a two year lunar mission, SCD devices are found to be fully operational, exhibiting an increase in leakage current proportional to received dose $(\sim 60 \%$ increase after $7.5 \times 10^{8}$ protons. $\mathrm{cm}^{-2}$ at $\left.-20{ }^{\circ} \mathrm{C}\right)$. Spectral resolution of $<400 \mathrm{eV} \mathrm{FWHM}$ at $\mathrm{Mn}-\mathrm{K} \alpha$ is achievable after a dose of $7.5 \times 10^{8}$ protons.cm ${ }^{-2}$ when operating at $-20{ }^{\circ} \mathrm{C}$, decreasing to $<350 \mathrm{eV}$ at $-40{ }^{\circ} \mathrm{C}$. This emphasises the need for cold operation

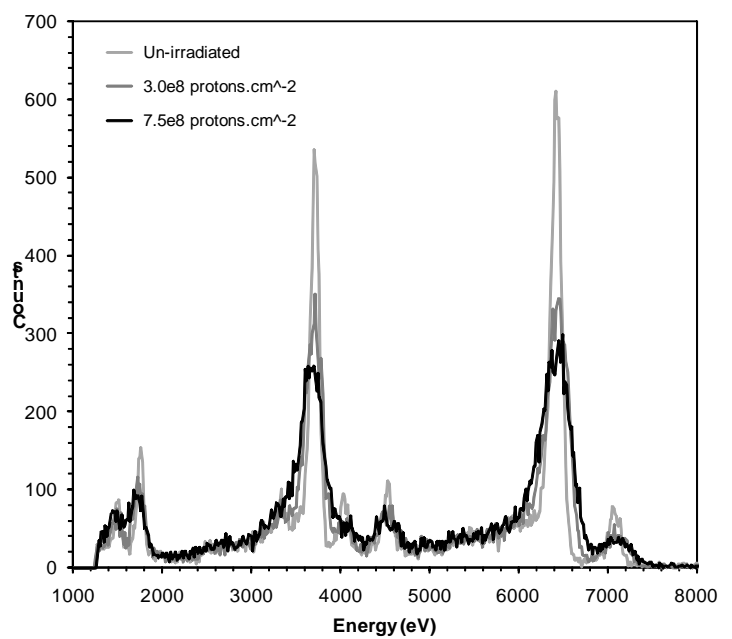

Fig. 4. Degradation of measured lunar regolith simulant (JSC-1A) spectrum following proton irradiation.

of SCDs for optimum performance in radiation environments.

\section{Acknowledgments}

The authors would like to thank Chris Howe at STFC Rutherford Appleton Laboratory for provision of the SCD modules used in the irradiation studies, Christian Erd at ESA for his comments and support, Katie Joy and Shoshana Weider at Birkbeck College for providing the lunar regolith simulant, ESA for funding the KVI proton beam facility time and the staff at KVI and the University of Birmingham for their support during proton irradiation tests.

\section{References}

[1] C. J. Howe et al., submitted to Planetary and Space Science (2008).

[2] D. R. Smith, J. Gow, A. D. Holland, Nuc. Inst. Meth. A583 (2007) 270.

[3] A.D. Holland, I.B. Hutchinson, D.R. Smith, P. Pool, Nuc. Inst. Meth. A521 (2004) 393.

[4] J. Gow, D. R. Smith, A. D. Holland, B. Maddison, C. Howe, P. Sreekumar, J. Huovelin, M. Grande, Proc. SPIE 6686 (2007).

[5] M. Grande et al., Planetary and Space Science 51 (2003) 427.

[6] M. Grande et al., Planetary and Space Science 55, (2007) 494.

[7] D. Heynderickx, B. Quaghebeur, E. Speelman, E. J. Daly, Proc. AIAA 0371 (2000). 